\title{
Synergistic Extraction of Manganese(II) and Iron(II) with Monothiothenoyltrifluoroacetone and 1,10-Phenanthroline
}

\author{
Hiroshi Kawamoto, Tsunemi Aiba and Hideo Akaiwa \\ Department of Applied Chemistry, Faculty of Engineering, Gunma University, Kiryu, Gunma 376
}

\begin{abstract}
The synergistic extraction of manganese(II) and iron(II) with monothiothenoyltrifluoroacetone(STTA=HR) and 1,10phenanthroline (phen=S) was examined. The main species extracted into chloroform phase were found to be $\mathrm{MnR}_{2} \mathrm{~S}$ and Fe $\mathrm{R}_{2} \mathrm{~S}_{2}$. This extraction system was applied to the quantitative separation of iron(II) from manganese(II). By the aid of phen, the extraction curves for both cations could be shifted to a lower $\mathrm{pH}$ region without losing the specificity of STTA toward iron(II).
\end{abstract}

Keywords Synergistic extraction, monothiothenoyltrifluoroacetone, 1,10-phenanthroline, manganese(II), iron(II)

Chaston et al. were the first to prepare monothiothenoyltrifluoroacetone(STTA) as well as its nickel(II) chelate. They showed that STTA acts as a ligand.' Thereafter, various metal-STTA complexes were prepared ${ }^{2,3}$, and STTA was then introduced into the field of solvent extraction. ${ }^{4}$ This reagent was applied to the spectrophotometric determination of mercury(II) ${ }^{5}$, and other elements. ${ }^{6}$ Although a synergistic extraction system of metal-STTA was studied $^{7}$ and applied to the spectrophotometric determination of iron(II) $)^{8,9}$, only a few works ${ }^{10}$ aiming the separation of metal ions have been reported.

In the present work, the synergistic effect of 1,10phenanthroline(phen) on the extraction of manganese(II) and iron(II) with STTA was studied. The result was applied to the separation of iron(II) from manganese(II).

\section{Experimental}

\section{Apparatus}

A Hitachi Model 170-30 atomic absorption spectrometer equipped with manganese and iron hollow cathode lamps (Hamamatsu Photonics) was used. A Horiba Model F-80 pH analyzer and a Iwaki KM type shaker were also used.

\section{Reagents}

STTA and phen were obtained from Wako Pure Chemicals, and were used without further purification. Chloroform and all the other chemicals used were of guaranteed grade. Deionized water was used throughout.

\section{Extraction procedure}

Twenty cubic centimeters of an aqueous solution containing $2.00 \times 100^{-5} \mathrm{~mol} \mathrm{dm}^{-3}$ manganese(II) or iron(II), $1.5 \%$ hydroxylamine sulfate and $0.1 \mathrm{~mol} \mathrm{dm}^{-3}$ sodium sulfate, whose $\mathrm{pH}$ was preliminarily adjusted by adding sulfuric acid or sodium hydroxide, was placed in a separatory funnel. An equal volume of chloroform solution containing STTA and phen was added; the mixture was then shaken vigorously for 10 min. After the phases were allowed to separate, manganese(II) or iron(II) remaining in the aqueous phase was determined with the atomic absorption spectrometer, and the $\mathrm{pH}$ was also measured. All experiments were carried out at room temperature (ca. $293 \mathrm{~K})$. The distribution ratio of a metal(II) was defined as follows:

$$
D=\frac{\text { The concentration of a metal in the } \mathrm{CHCl}_{3} \text { phase }}{\text { The concentration of a metal in the } \mathrm{H}_{2} \mathrm{O} \text { phase }} \text {. }
$$

\section{Theoretical}

By assuming that the main species extracted with STTA (HR) and phen (S) is $M_{2} S_{n}(n=0,1,2)$, the extraction reaction can be expressed by

$$
\mathrm{M}^{2+}+2 \mathrm{HR}_{\text {org }}+n \mathrm{SS}_{\text {org }} \rightleftarrows \mathrm{MR}_{2} \mathrm{~S}_{n, \text { org }}+2 \mathrm{H}^{+}, \ldots
$$

where $\mathrm{M}^{2+}$ represents manganese(II) and iron(II), and the subscript org denotes the organic phase. The extraction constant is defined by Eqn (3). Strictly speaking, the species remaining in the aqueous phase should be expressed as $\mathrm{MR}_{i} \mathrm{~S}_{j^{2-i}}(i, j=0-3)$, for which the formation reaction and the equilibrium constant are written by Eqns (4) and (5), respectively. 


$$
\begin{aligned}
& K_{\mathrm{ex}, n}=\frac{\left[\mathrm{MR}_{2} \mathrm{~S}_{n}\right]_{\mathrm{org}}\left[\mathrm{H}^{+}\right]^{2}}{\left[\mathrm{M}^{2+}\right][\mathrm{HR}]_{\mathrm{org}}^{2}[\mathrm{~S}]_{\mathrm{org}}^{n}} \\
& \mathrm{M}^{2+}+i \mathrm{HR}_{\mathrm{org}}+j \mathrm{~S}_{\mathrm{org}} \rightleftarrows \mathrm{MRS}_{f^{2-}}-i+i \mathrm{H}^{+} \quad \ldots \\
& K_{i, j}=\frac{\left[\mathrm{MR}_{i} \mathrm{~S}_{j}^{2-}\right]\left[\mathrm{H}^{+}\right]^{i}}{\left[\mathrm{M}^{2+}\right][\mathrm{HR}]_{\mathrm{org}}^{i}[\mathrm{~S}]_{\mathrm{org}}^{j}}
\end{aligned}
$$

Then, the distribution ratio given by Eqn (1) can be written as

$$
D=\frac{\sum_{n}\left[\mathrm{MR}_{2} \mathrm{~S}_{\mathrm{a}}\right]_{\mathrm{org}}}{\sum_{i} \sum_{j}\left[\mathrm{MR} \mathrm{R}_{j}^{2-\eta}\right]}
$$

From Eqns (3), (5) and (6), we obtain

$$
D=\frac{[\mathrm{HR}]_{\text {ors }}^{2} \sum_{n} K_{\mathrm{ex,n}, n}[\mathrm{~S}]_{\mathrm{org}}^{n}}{\left[\mathrm{H}^{+}\right]^{2} \sum_{i} \sum_{j} K_{j, j}[\mathrm{HR}]_{\mathrm{org}}^{l}[\mathrm{~S}]_{\text {org }}^{j}\left[\mathrm{H}^{+}\right]^{-i}} .
$$

By neglecting the concentration of phen combined with a metal(II), the total phen concentration added to the extraction system is given by

$$
\begin{aligned}
\mathbf{S S}_{\text {tot }} & =[\mathbf{S}]_{\text {ors }}+[\mathbf{S}]+\left[\mathbf{H S}^{+}\right] \\
& =[\mathbf{S}]_{\text {ors }}\left(1+\frac{1}{\boldsymbol{K}_{\mathrm{DS}}}+\frac{\left[\mathbf{H}^{+}\right]}{\boldsymbol{K}_{\mathbf{D S} K_{\mathbf{H S}}}}\right)
\end{aligned}
$$

where $K_{\mathrm{DS}}=[\mathrm{S}]_{\mathrm{org}}[\mathrm{S}]^{-1}$ and $K_{\mathrm{HS}}=\left[\mathrm{H}^{+}\right][\mathrm{S}]\left[\mathrm{HS}^{+}\right]^{-1}$.

Introducing the values $\log K_{\mathrm{DS}}=3.05$ and $\mathrm{p} K_{\mathrm{HS}}=5.05^{11}$, Eqn (8) can be expressed as

$$
[S]_{\text {tot }}=[S]_{\text {org }}\left(1.00+100\left[H^{+}\right]\right)
$$

The total concentration of STTA can be written by Eqn (10), if the concentration of STTA combined with a metal(II) can be neglected compared with that of free STTA:

$$
\begin{aligned}
{\left[\mathrm{HR}_{\text {tot }}\right.} & =[\mathrm{HR}]_{\text {org }}+[\mathrm{HR}]+\left[\mathrm{R}^{-}\right] \\
& =[\mathrm{HR}]_{\text {org }}\left(1+\frac{1}{K_{\mathrm{DR}}}+\frac{K_{\mathrm{HR}}}{K_{\mathrm{DR}}\left[\mathrm{H}^{+}\right]}\right) \cdots
\end{aligned}
$$

where $K_{\mathrm{DR}}=[\mathrm{HR}]_{\mathrm{org}}[\mathrm{HR}]^{-1}$ and $K_{\mathrm{HR}}=\left[\mathrm{H}^{+}\right]\left[\mathrm{R}^{-}\right][\mathrm{HR}]^{-1}$. By using $\log K_{\mathrm{DR}}=4.19$, which was obtained by Uhlemann and Mueller's method, and $\mathrm{p} K_{\mathrm{HR}}=4.10,12$ Eqn (10) can be rewritten as

$$
[\mathrm{HR}]_{\text {tot }}=[\mathrm{HR}]_{\mathrm{org}}\left(1.00+\frac{5.12 \times 10^{-9}}{\left[\mathrm{H}^{+}\right]}\right)
$$

Equations (9) and (11) enable us to calculate [S] $]_{\text {org }}$ and [HR] $]_{\text {org, }}$ respectively.

\section{Results and Discussion}

The extraction of manganese(II)

If the presence of phen causes the formation of $\mathrm{MnR}_{2} \mathrm{~S}$ and if the main species remaining in the aqueous phase is $\mathrm{Mn}^{2+}$, Eqn (7) can be written as

$$
\log D=\log \left(K_{\text {ex }, 0}+K_{\text {ex, }, 1}[\mathrm{~S}]_{\text {org }}\right)+2 \log [H R]_{\text {org }}+2 \mathrm{pH} .
$$

In the case that the above assumption is valid, a plot of $\log D$ against $\mathrm{pH}$ keeping [S$]_{\text {org }}$ and [HR] $]_{\text {org }}$ constant will give a straight line with a slope of 2 . As seen in Fig. 1, plots at a pH higher than 6 fall on a straight line

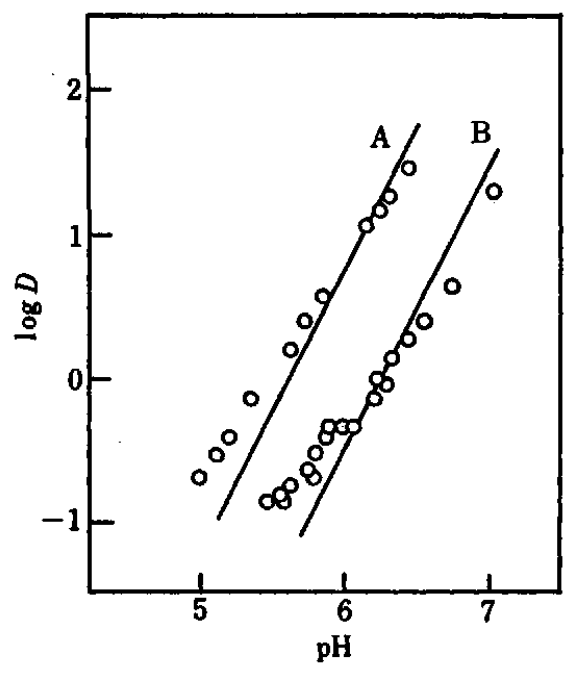

Fig. 1 Plots of $\log D(\mathrm{Mn})$ against pH. STTA, $1.00 \times 10^{-3}$ M; phen, A, $2.00 \times 10^{-3} \mathrm{M} ; \mathrm{B}, 2.00 \times 10^{-4} \mathrm{M}$.

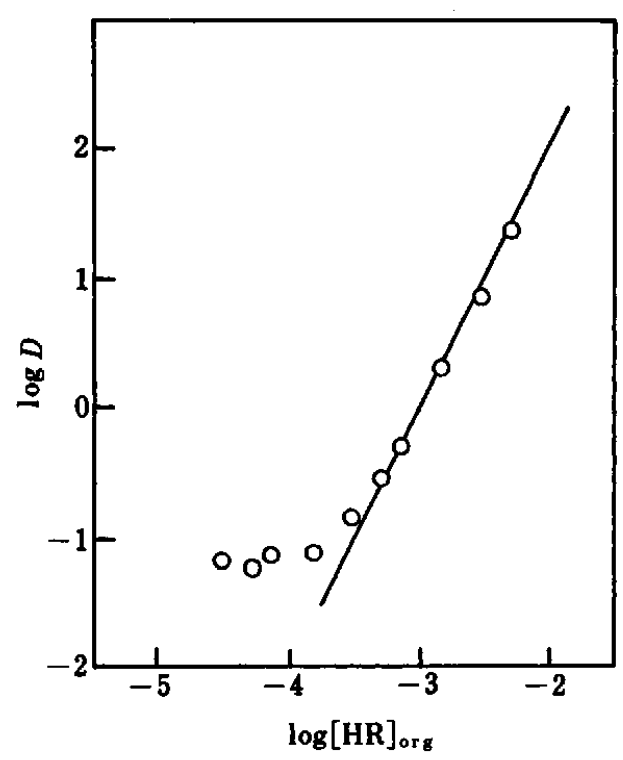

FIg. 2 Logarithmic plot of $D(\mathrm{Mn})$ against STTA concentration. Phen, $2.00 \times 10^{-3} \mathrm{M} ; \mathrm{pH} 5.45$. 
having a slope of 2 , although positive deviations due to unknown reasons are seen at the low $\mathrm{pH}$ region. Figure 2 shows a straight line whose slope is 2 , indicating that Eqn (13) is valid. However, the reason for the positive deviation in the region of $\log [H R]_{\text {org }}<-$ 3.5 is still unknown. Equation (13) can be rewritten as

$$
\log D=\log \frac{K_{\mathrm{ex}, 0}[\mathrm{HR}]_{\mathrm{org}}^{2}}{\left[\mathrm{H}^{+}\right]^{2}}+\log \left(1+\frac{K_{\mathrm{ex}, 1}[\mathrm{~S}]_{\mathrm{org}}}{K_{\mathrm{ex}, 0}}\right)
$$

Equation (14) can be expressed by a normalized curve (15), by taking $X=K_{\text {ex, } 1}[S]_{\text {org }} K_{\text {ex, }}^{-1}$ and $Y=\log D-\log$ $K_{\text {ex, } 0}[\mathrm{HR}]_{\text {org }}^{2}\left[\mathrm{H}^{+}\right]^{-2}$.

$$
Y=\log (1+X)
$$

As a result of curve-fitting, in which our data well fits Eqn (15) (Fig. 3), $\log K_{\text {ex }, 0}=-5.82$ and $\log K_{\text {ex }, 1}=-2.37$ were obtained.

\section{The extraction of iron $(I)$}

By assuming that the main complex in the organic phase is $\mathrm{FeR}_{2} \mathrm{~S}_{2}$, and that the species remaining in the aqueous phase are $\mathrm{FeS}_{3}{ }^{2+}$ and $\mathrm{FeRS}_{2}{ }^{+}$, Eqn (7) can be rewritten as

$$
D=\frac{[\mathrm{HR}]_{\mathrm{org}}^{2} K_{\mathrm{ex}, 2}}{\left[\mathrm{H}^{+}\right]^{2}\left(K_{0,3}[\mathrm{~S}]_{\mathrm{org}}+K_{1,2}[\mathrm{HR}]_{\mathrm{org}}\left[\mathrm{H}^{+}\right]^{-1}\right)}
$$

When the predominant species in the aqueous phase is $\mathrm{FeRS}_{2}{ }^{+}$, Eqn (16) approximates to Eqn (17):

$$
\log D=\log \frac{K_{\mathrm{ex}, 2}}{K_{1,2}}+\log [\mathrm{HR}]_{\mathrm{org}}+\mathrm{pH}
$$

Equation (17) suggests that a plot of $\log D$ against pH keeping [HR $]_{\text {org }}$ constant will give a straight line having a slope of unity, which agrees with the plot shown in Fig.

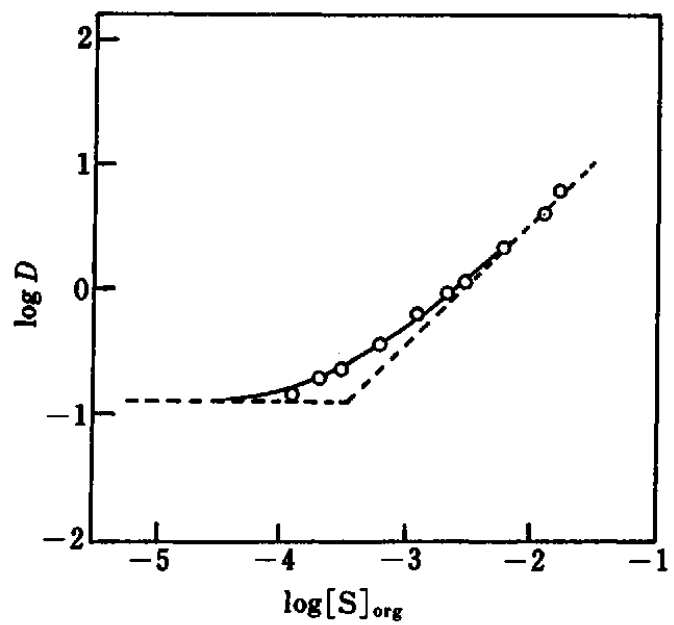

Fig. 3 Logarithmic plot of $D(\mathrm{Mn})$ against phen concentration. - , the best fitted curve; STTA, $1.00 \times 10^{-3} \mathrm{M} ; \mathrm{pH} 5.46$.
4. On the other hand, Eqn (16) can be rewritten as

$$
\begin{aligned}
\log D-2 \log [\mathrm{HR}]_{\text {org }} & =\log \frac{K_{\text {ex, }, 2}}{\left[\mathrm{H}^{+}\right]^{2}}-\log K_{0,3}[\mathrm{~S}]_{\text {org }} \\
& -\log \left(1+\frac{K_{1,2}[\mathrm{HR}]_{\text {org }}}{K_{0,3}[\mathrm{~S}]_{\text {org }}\left[\mathrm{H}^{+}\right]}\right) .
\end{aligned}
$$

By taking $Y=\log D-2 \log [\mathrm{HR}]_{\text {org }}-\log K_{\text {ex }, 2}\left[\mathrm{H}^{+}\right]^{-2}+\log$ $K_{0.3}[\mathrm{~S}]_{\text {org }}$ and $X=K_{1,2}[\mathrm{HR}]_{\text {org }} K_{0,3}^{-1}[\mathrm{~S}]_{\text {org }}^{-1}\left[\mathrm{H}^{+}\right]^{-1}$, Eqn (18) can be expressed by a normalized curve (19):

$$
Y=-\log (1+X)
$$

As seen in Fig. 5, the plots are located on the normalized curve, indicating that Eqn (18) is available. In the case that $K_{0,3}[\mathrm{~S}]_{\mathrm{org}} \gg K_{1,2}[\mathrm{HR}]_{\text {org }}\left[\mathrm{H}^{+}\right]^{-1}$, Eqn (20) is obtained from Eqn (16):

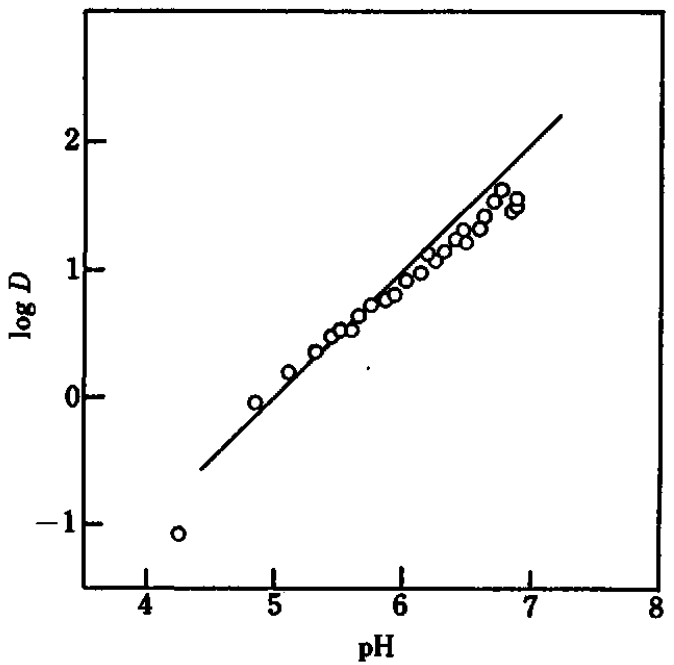

Fig. 4 Plots of $\log D(\mathrm{Fe})$ against pH. STTA, $1.00 \times 10^{-3} \mathrm{M}$; phen, $2.00 \times 10^{-3} \mathrm{M}$.

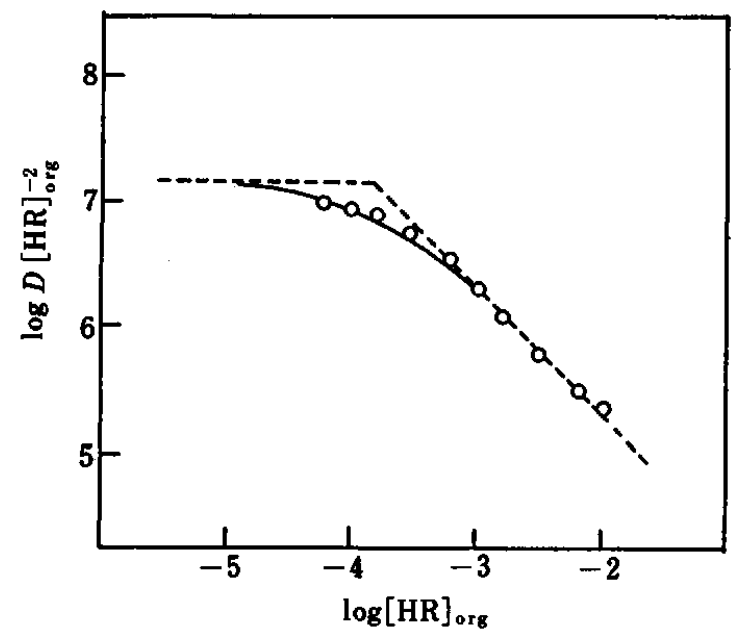

Fig. 5 Effect of STTA concentration on the extraction of iron(II). -, the best fitted curve; phen, $2.00 \times 10^{-3} \mathrm{M} ; \mathrm{pH} 5.10$. 


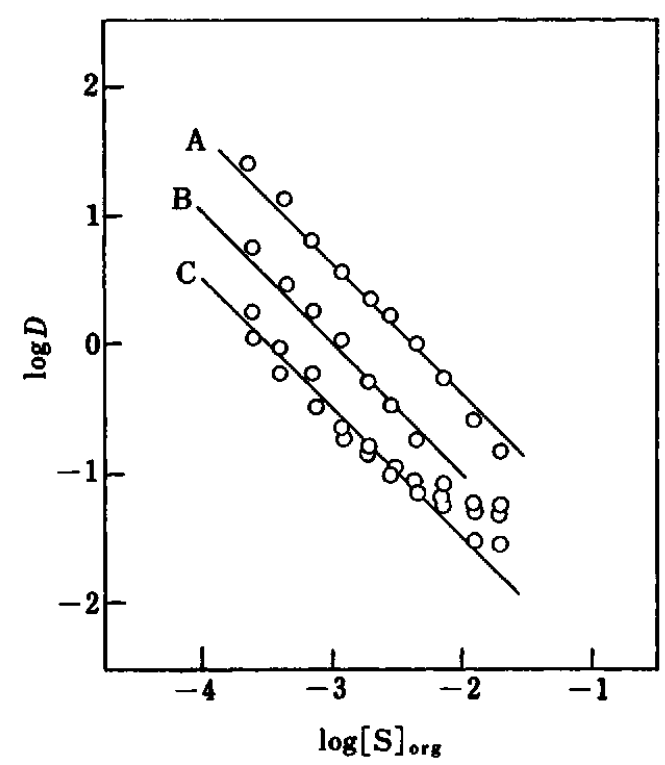

Fig. 6 Logarithmic plots of $D(\mathrm{Fe})$ against phen concentration. Plot-STTA/M-pH: A-1.00 $\times 10^{-3}-5.19 ; \quad$ B-2.00X $10^{-3}-4.57 ; \mathrm{C}-1.00 \times 10^{-4}-5.20$.

$$
\log D=\log \frac{K_{\text {ex, }, 2}}{K_{0,3}}+2 \log [\mathrm{HR}]_{\text {org }}+2 \mathrm{pH}-\log [\mathrm{S}]_{\text {org }} \text {. }
$$

In Fig. 6, a plot of $\log D$ against $\log [\mathrm{S}]_{\text {org }}$ keeping [HR] org and $\mathrm{pH}$ constant is shown by a straight line having a slope of minus unity, as predicted by Eqn (20). All the above results indicate that Eqn (16) is applicable to the present system; $\log K_{\mathrm{ex}, 2} K_{0,3}^{-1}=-5.75, \log$ $K_{\text {ex. } 2} K_{1,2}^{-1}=-1.77$, and $\log K_{1,2} K_{0.3}^{-1}=-3.98$ were obtained. Since $\log \left[\mathrm{FeS}_{3}{ }^{2+}\right]\left[\mathrm{Fe}^{2+}\right]^{-1}[\mathrm{~S}]^{-3}=-21.3,{ }^{13} \log K_{\text {ex }, 2}=6.40$ and $\log K_{1,2}=8.17$ could be estimated.

\section{The separation of iron(II) from manganese(II)}

The solid curves given in Fig. 7-B and 7-C for iron(II) were obtained by calculations using Eqn (20), which can be fitted well to the experimental results. However, the extraction curves estimated from Eqn (13) deviate from those obtained by experiments, and the degree of the deviation becomes larger at higher $\mathrm{pH}$ region, probably because of hydrolysis of manganese(II).

Although the separation of iron(II) from manganese(II) could be performed without the aid of phen (Fig. 7-A), the $\mathrm{pH}$ condition for quantitative separation was an alkaline medium, $\mathrm{ca}$. $\mathrm{pH} 7.5$, where the troublesome hydrolysis of metal ions might occur, as mentioned above. On the contrary, iron(II) could be separated quantitatively from manganese(II) at a slightly acidic region, $\mathrm{ca}$. $\mathrm{pH} 5.0$, by the addition of a small amount of phen (Fig. 7-B,C). The addition of the synergist shifts the extraction curves to lower $\mathrm{pH}$ region without losing the specificity of STTA toward iron(II).

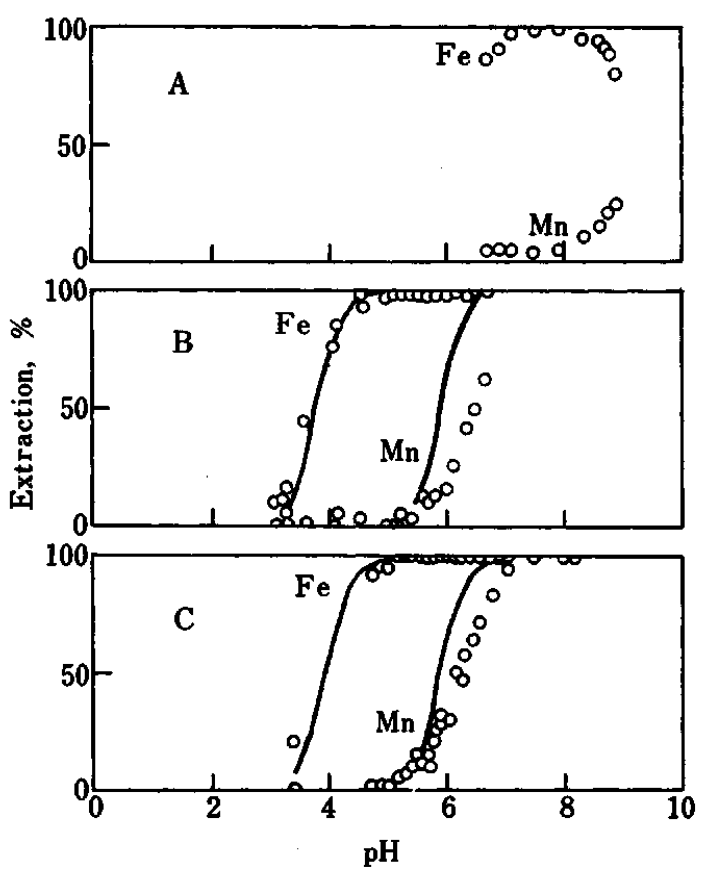

Fig. 7 Extraction curves. Shaking time, $30 \mathrm{~min}$; STTA, $1.00 \times 10^{-3} \mathrm{M}$. Phen: A, none; B, $1.00 \times 10^{-4} \mathrm{M} ; \mathrm{C}, 2.00 \times 10^{-4}$ $\mathrm{M}$.

This work was supported in part by the Grant-in-Aid for Scientific Research No. 61470035, Ministry of Education, Science and Culture.

\section{References}

1. S. H. H. Chaston, S. E. Livingstone, T. N. Lockyer, V. A. Pickeles and J. S. Shannon, Aust. J. Chem.,18, 673 (1965).

2. E. W. Berg and K. P. Reed, Anal. Chim. Acta, 36, 372 (1966)

3. R. K. Y. Ho, S. E. Livingstone, and T. N. Lockyer, Aust. J. Chem., 19, 1179 (1966).

4. V. M. Shinde and S. M. Khopkar, Chem. Ind., 1967, 1785.

5. J. Saika and H. Komuro, Bunseki Kagaku, 19, 1214 (1970).

6. T. Honjo and T. Kiba, Bunseki Kagaku, 31, 676 (1972).

7. T. Honjo, S. Yashima, and T. Kiba, Bull. Chem. Soc. Jpn., 46, 3772 (1973).

8. M. Chikuma, A. Yokoyama, Y. Ooi and H. Tanaka, Chem. Pharm. Bull., 23, 507 (1975).

9. T. Honjo, Y. Fujioka, H. Itoh and T. Kiba, Anal. Chem., 49, 2241 (1977).

10. T. Honjo, T. Unemoto and T. Kiba, Bunseki Kagaku, 23, 203 (1974).

11. C. W. Woodward and H. Freiser, Anal. Chem., 40, 345 (1968).

12. E. Uhlemann and H. Mueller, Z. Chem., 8, 185 (1968).

13. G. Anderegg, Helv. Chim. Acta, 46, 2397 (1963).

(Received May 28, 1987)

(Accepted June 5, 1987) 